\begin{tabular}{|c|c|c|c|c|c|c|c|}
\hline Nr. & $\begin{array}{c}\text { Durch- } \\
\text { musterung }\end{array}$ & \multicolumn{2}{|l|}{$\alpha$ I9II.0 } & \multicolumn{2}{|c|}{81911.0} & $E_{p}$. & Anscl \\
\hline 162 & $65^{\circ} I 54^{8}$ & $2 I^{\mathrm{h}} \quad 7^{\mathrm{m}_{2} 8.2}$ & & $+66^{\circ}$ & $I^{\prime}$ I 5.8 & $\begin{array}{l}1890+ \\
1.79\end{array}$ & Bo V \\
\hline 63 & I 34688 & 2 I 1857.1 & & +135 & $5443 \cdot 1$ & 1.55 & \\
\hline 64 & 65 I 577 & 21 I 923.2 & & +655 & $54 \quad 48.2$ & 1.79 & Chri \\
\hline 165 & $65 \times 579$ & $\begin{array}{lll}21 & 20 & 8.5\end{array}$ & & +655 & 583.8 & 1.79 & \\
\hline 166 & I 34701 & $\begin{array}{lll}2 \text { I } & 20 & 59.9\end{array}$ & & $+13:$ & $35 \quad 39.8$ & 1.55 & \\
\hline 167 & - & 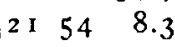 & & $+2 \times 3$ & $38 \quad 16.9$ & 1.60 & \\
\hline 163 & - & $\begin{array}{lll}23 & 9 & 5 \mathrm{I} .7\end{array}$ & & +143 & $3^{2} \quad 5^{2.3}$ & I. $5^{8}$ & Lpz I \\
\hline 169 & $65 \times 44$ & $\begin{array}{lll}23 & 16 & 19.0 \\
23 & 16 & 26.8\end{array}$ & 36 & $\begin{array}{l}+62 \\
+62\end{array}$ & $\begin{array}{ll}8 & 40.6 \\
27 & 19.1\end{array}$ & I. 58 & \\
\hline 70 & $55^{164}$ & $\begin{array}{lll}23 & 19 & 49.7\end{array}$ & & +53 & 3325.5 & 1.58 & $»$ \\
\hline & Größe & senale & & $\mathrm{n}$ im & yste & Ite & I D D D \\
\hline & $\begin{array}{l}\text { Nr. Gr. } \\
29^{m} \cdot I\end{array}$ & $\begin{array}{cc}\text { Nr. } & \text { Gr. } \\
18 & 9^{\mathrm{m}} \cdot 4\end{array}$ & & $\begin{array}{l}\mathrm{Gr} \\
9^{\mathrm{m}} \cdot 5\end{array}$ & $\begin{array}{ll}\mathrm{Nr} & \\
45 & 9\end{array}$ & & $\begin{array}{cc}\text { Nr. } & \text { Gr } \\
55 & 9^{\text {m }}\end{array}$ \\
\hline & 49.5 & $24 \quad 9.6$ & 35 & 9.5 & 46 & 9. I & 569. \\
\hline & 59.5 & $25 \quad 9.2$ & 36 & $9 \cdot 3$ & & 0.8 & 57 \\
\hline & 69.5 & $26 \quad 9.4$ & 37 & $9 \cdot 5$ & I I & & 58 \\
\hline & 79.5 & $28 \quad 9.4$ & $3^{8}$ & 9. I & 48 & $9 . \mathrm{I}$ & 599. \\
\hline & $8 \quad 9.5$ & $29 \quad 9.3$ & 40 & 9.6 & 49 & $9 \cdot 3$ & 60 \\
\hline & 99.2 & $3 \circ 9.2$ & 41 & 9.4 & 50 & 8.9 & $6 \mathrm{I}$ \\
\hline & $10 \quad 9.6$ & 319.2 & 42 & 9.4 & $5^{2}$ & $9 \cdot 3$ & 62 \\
\hline & I3 9.4 & $329 \cdot 3$ & 43 & 9.4 & 53 & $9 \cdot 3$ & 63 \\
\hline & I 49.3 & $33 \quad 9 \cdot 3$ & 44 & 9.5 & 54 & 9.6 & 669 \\
\hline
\end{tabular}

Bergedorf, Sternwarte, I 9 I 2 Dezember.

\begin{tabular}{|c|c|c|c|c|c|c|c|c|c|}
\hline $\begin{array}{l}\mathrm{Nr} . \\
67\end{array}$ & $\begin{array}{c}\mathrm{Gr} . \\
\mathrm{I}^{\mathrm{m}} \cdot 5\end{array}$ & $\begin{array}{l}\text { Nr. } \\
87\end{array}$ & $\begin{array}{l}\text { Gr. } \\
9^{\mathrm{m}} \cdot 3\end{array}$ & $\begin{array}{l}\text { Nr. } \\
\text { I } \circ 9\end{array}$ & $\begin{array}{l}\text { Gr. } \\
9^{m} \cdot 5\end{array}$ & $\begin{array}{l}\text { Nr. } \\
\text { I } 30\end{array}$ & $\begin{array}{l}-\mathrm{Gr} . \\
9^{\mathrm{m}} \cdot 3\end{array}$ & $\begin{array}{l}N_{r} \\
154\end{array}$ & $\begin{array}{c}\text { Gr. } \\
8^{\mathrm{mn}} \cdot 7\end{array}$ \\
\hline 68 & 9.7 & 90 & 9.I & 110 & $9 \cdot 3$ & I 31 & 9.6 & 155 & 9.2 \\
\hline 69 & 9.7 & $9 \mathrm{I}$ & 9.2 & I I I & 9.4 & 132 & 9.5 & $15^{6}$ & 9.1 \\
\hline 70 & $9 \cdot 3$ & 92 & 9.4 & I I 2 & 9.4 & 133 & 9.6 & I 57 & \\
\hline 71 & $9 \cdot 3$ & 93 & I Odpl. & I I 4 & 9.6 & 134 & 9.1 & 158 & \\
\hline 72 & $9 \cdot 3$ & 94 & 9.6 & 115 & 9.6 & I 35 & $9 \cdot 3$ & I 59 & 9. \\
\hline 73 & $9 \cdot 3$ & 95 & 9.8 & 116 & 9.2 & I 38 & 9.5 & I6 I & \\
\hline 74 & 9.2 & 96 & 9.3 & 117 & $9 \cdot 3$ & I 39 & 9.5 & 162 & \\
\hline 75 & $9 \cdot 3$ & 97 & I I & I 8 & 0.3 & 140 & 9.5 & 163 & 10. \\
\hline 76 & 9.5 & 98 & 9.4 & 119 & 9.5 & $14 \mathrm{r}$ & 9.5 & 164 & \\
\hline 77 & 9.6 & 99 & $9 \cdot 3$ & 120 & 9.5 & 142 & $9 \cdot 3$ & 165 & 9. \\
\hline 78 & 9.5 & 100 & 9.2 & 12 I & 9.9 & I 43 & 9.2 & 166 & \\
\hline 79 & 9.4 & 101 & 9.2 & 122 & 9.8 & I 45 & 9.4 & 167 & \\
\hline 80 & 9.5 & 102 & 9.4 & 123 & I 0.2 & 146 & 9.6 & 168 & \\
\hline $8 \mathrm{I}$ & 9.6 & 103 & $9 \cdot 5$ & 124 & $9 \cdot 3$ & I 47 & $9 \cdot 5$ & & IO. \\
\hline 82 & 9.4 & 104 & 9.5 & 125 & 9.2 & I 48 & $9 \cdot 3$ & & \\
\hline $8_{3}$ & 9.4 & 105 & $9 \cdot 3$ & 126 & 9.2 & I 49 & 9.5 & 170 & \\
\hline 84 & 9.4 & 106 & $9 \cdot 3$ & 127 & 9.2 & I $5 \mathrm{I}$ & 10.1 & & \\
\hline 85 & $9 \cdot 3$ & 107 & $9 \cdot 3$ & 128 & 9.4 & I 52 & 9.4 & & \\
\hline 86 & 9.5 & I08 & 9.5 & 129 & 9.5 & I 53 & 9.2 & & \\
\hline
\end{tabular}

Die obigen Anschlüsse sind meist Revisionen von Sternen des Rümkerkataloges. Mit Ausnahme der Nummern 1 7, 19-23 und 39 sind sie von $K$. Graff ausgeführt. Alle Kontrollrechnungen, sowie die Zusammenstellung der Beobachtungen für den Druck hat Dr. Bottlinger ausgeführt.

\title{
Mitteilungen über BD-Sterne.
}

Due stelle $\mathrm{BD}-0^{\circ} 3946$ e $395^{2}$ che ora mancano in Cielo.

\begin{tabular}{|c|c|c|c|c|c|c|}
\hline \multirow[t]{2}{*}{$*$} & \multirow[t]{2}{*}{ Gr. } & \multicolumn{2}{|c|}{$\alpha 1855$} & $\delta 1855$ & \multicolumn{2}{|l|}{$\mathrm{BD}$} \\
\hline & & $\left(20^{\mathrm{h}} 6\right.$ & $35^{\mathrm{s}} \cdot 2$ & $\left(\begin{array}{lll}-0^{\circ} & 2 & 7 \\
\end{array}\right.$ & $-0^{\circ} 3946$ & $9^{m} \mathrm{I}$ \\
\hline I & $9^{m} \cdot 5$ & 206 & 47.8 & $-0 \quad 27.5$ & -0.3947 & 9.2 \\
\hline 2 & 9.5 & 206 & 50.6 & -0 27.8 & -03948 & $9 \cdot 5$ \\
\hline Cf. & 8.3 & 206 & 53.6 & -o I 7.4 & -03949 & 8.3 \\
\hline 3 & $9 \cdot 5$ & 206 & $5^{8 \cdot 3}$ & $\begin{array}{ll}-0 & 26.3\end{array}$ & $-\circ 395^{\circ}$ & $9 \cdot 3$ \\
\hline 4 & I 2 & 207 & I.O & $-0 \quad 28.4$ & -- & - \\
\hline 5 & I 2 & 207 & $3 \cdot 4$ & - $0 \quad 23.9$ & - & 一 \\
\hline 6 & $9 \cdot 5$ & 207 & 5.0 & $-0 \quad 23.2$ & $-\circ 395^{1}$ & 9.5 \\
\hline & & $(207$ & $9 \cdot 3$ & $\left(\begin{array}{ll}-0 & 30.5\end{array}\right)$ & $-0395^{2}$ & $9 \cdot 5$ \\
\hline 7 & 9.5 & 207 & 14.9 & -021.5 & - o 3953 & 9.4 \\
\hline 8 & 9.5 & 207 & 19.2 & -o 23.6 & $-\circ 3954$ & $9 \cdot 5$ \\
\hline
\end{tabular}

La sera del 7 Dicembre r 9 r 2 osservando la cometa I9ı2 c (Borrelly) colla stella Cf. $=-0^{\circ} 3949$, e non corrispondendomi nel campo del cannocchiale la configurazione della carta BD No. I I, passai in rassegna le stelline prossime a Cf. assicurandomi che in cielo ne mancavano due di quelle segnate sulla carta. Di poi nella stessa sera, e nella successiva, presi le differenze fra Cf. e le otto stelline segnate coi numeri I ad 8 nella prima colonna della superiore tabella. Questa fu composta colle suddette differenze, e colla posizione BD 1855 della stella Cf. In tal modo restarono identificate tutte le stelle che le sono prossime meno le due mancanti 3946 e $395^{2}$ che nella tabella sono indicate colla posizione BD scritta frá parentesi. Nei limiti della precisione $B D$ potrebbe essere che alla $395^{2}$ corrispondesse la 4 che attualmente è di $12^{\mathrm{m}}$ circa.

La stella $+\mathrm{I}^{\circ} 42048^{\mathrm{m}} \cdot 7=\mathrm{AG}$ Alb $699 \mathrm{r}$ porta erroneamente in questo catalogo l'indicazione di duplicità, che va invece attribuita giustamente alla stella che precede boreale; $\mathrm{da}$ indicarsi quindi con $6990^{6}$. Questa è quella che fu vista doppia conforme la nota del catalogo, all'equatoriale di Amici, la sera del 4 Dicembre I9 I2, osservando la cometa I 9 I 2 c (Borrelly).

Arcetri, 19 I 2 Dic. I 2.

A. Abcti

Todesanzeige. Der Direktor der Sternwarte in Santiago, Ch., Friedrich Wilhelm Ristenpart ist Anfang April d. J. aus dem Leben geschieden. Eine Würdigung der Lebensarbeit des um die Astronomie hochverdienten Gelehrten kann erst nach Eintreffen näherer Nachrichten von der Stätte seiner Wirksamkeit in den letzten Jahren erfolgen. $K$.

Inhalt zu Nr. 4647. K. Graff, H. Thiele. Beobachtungen von Kometen, Planeten und Fixsternen. 257. - A. Abetti. Mitteilungen uber BDSterne. $27 \mathrm{I}$. - Todesanzeige, $27 \mathrm{I}$. 